\title{
EFFECT OF PEEP AND TIDAL VOLUME ON AERATION OF THE PRETERM NEONATAL
} LUNG

K.I. Wheeler ${ }^{1,2,3,4}$, M.J. Kitchen ${ }^{5}$, M.J. Wallacce ${ }^{2}$, M.S. Islam ${ }^{5}$, M.L. Siew ${ }^{2}$, A. Fouras ${ }^{5}$, P.G. Davis ${ }^{4,6,7}$, C.J. Morley $^{6}$, R.A. Lewis ${ }^{5}$, S.B. Hooper ${ }^{2}$

${ }^{I}$ Newborn Research, Royal Women's Hospital, Melbourne, Parkville, ${ }^{2}$ Ritchie Center for Medical Research, Monash University, ${ }^{3}$ NMHRC CCRE in Newborn Medicine. RWH, ${ }^{4}$ Murdoch Childrens Research Institute,

${ }^{5}$ Monash University, ${ }^{6}$ Newborn Research, Royal Women's Hospital, ${ }^{7}$ University of Melbourne, Melbourne, VIC, Australia

Previous studies suggest that positive end expiratory pressure (PEEP) promotes aeration of the preterm lung. The role of tidal volume has been less clear. We aimed to study the effects of different PEEPs $(0,5,8$ and 10 $\left.\mathrm{cm} \mathrm{H}_{2} \mathrm{O}\right)$ and VTs $(4,8$, and $12 \mathrm{~mL} / \mathrm{kg}$ ) on the establishment of functional residual capacity (FRC).

$27 \mathrm{~d}$ preterm rabbit pups (equivalent to a surfactant deficient extremely preterm infant) were anaesthetised and surgically intubated. Volume targeted intermittent positive pressure ventilation (IPPV) was provided at each permutations of the above PEEPs and VTs. The primary outcome was FRC after 160 inflations, measured by plethysmography. Secondary outcomes included static compliance and homogeneity of tidal ventilation assessed using synchrotron based techniques (Spring8, Japan).

Mean (SE) FRC and static compliance after 160 inflations are shown below:

\begin{tabular}{|c|c|c|c|c|c|c|c|c|}
\hline & \multicolumn{4}{|c|}{ FRC } & \multicolumn{4}{|c|}{ Compliance } \\
\hline & 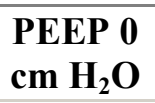 & $\begin{array}{l}\text { PEEP } 5 \\
\mathrm{~cm} \mathrm{H}_{2} \mathrm{O}\end{array}$ & 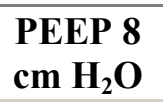 & $\begin{array}{l}\text { PEEP 10 } \\
\mathrm{cm} \mathrm{H} \mathrm{H}_{2} \mathrm{O}\end{array}$ & 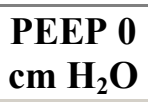 & $\begin{array}{l}\text { PEEP } 5 \\
\mathrm{~cm} \mathrm{H}_{2} \mathrm{O}\end{array}$ & $\begin{array}{l}\text { PEEP 8 } \\
\text { cm } \mathrm{H}_{2} \mathrm{O}\end{array}$ & 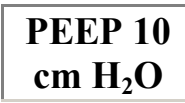 \\
\hline $\begin{array}{c}\text { VT } 4 \\
\mathrm{~mL} / \mathrm{kg}\end{array}$ & $2.4(1.1)$ & $3.0(0.4)$ & $2.0(0.4)$ & $3.3(0.6)$ & $\begin{array}{c}0.10 \\
(0.01)\end{array}$ & $\begin{array}{c}0.13 \\
(0.01)\end{array}$ & $\begin{array}{c}0.17 \\
(0.01)\end{array}$ & $0.23(0.02)$ \\
\hline $\begin{array}{c}\text { VT } 8 \\
\mathrm{~mL} / \mathrm{kg}\end{array}$ & $0.7(0.3)$ & $2.9(0.7)$ & $5.4(1.4)$ & $4.8(0.8)$ & $\begin{array}{c}0.18 \\
(0.01)\end{array}$ & $\begin{array}{c}0.24 \\
(0.02)\end{array}$ & $\begin{array}{c}0.31 \\
(0.01)\end{array}$ & $0.36(0.02)$ \\
\hline $\begin{array}{l}\text { VT } 12 \\
\mathrm{~mL} / \mathrm{kg}\end{array}$ & $1.4(0.5)$ & $2.8(0.9)$ & $5.2(0.9)$ & $9.6(2.1)$ & $\begin{array}{c}0.26 \\
(0.01)\end{array}$ & $\begin{array}{c}0.36 \\
(0.03)\end{array}$ & $\begin{array}{c}0.48 \\
(0.02)\end{array}$ & $0.57(0.01)$ \\
\hline
\end{tabular}

[Table]

Linear regression of both FRC and compliance was statistically significant against both VT and PEEP separately $(\mathrm{p} \leq 0.01)$. No pup developed a pneumothorax. Video imaging demonstrates more homogeneous tidal ventilation in pups with better established FRC.

PEEP and VT both played a role in the establishment of FRC in fully ventilated infants. Future studies should assess which strategies promote aeration at birth whilst minimising lung injury. 